\title{
Effects of hUCB-MSCs on recovery of neurological function and TERT expression in brain tissue of rats with cerebral ischemia-reperfusion injury
}

\author{
XIAOHUI HUANG ${ }^{1}$, SHUANGLI ZHANG ${ }^{2}$, FUCHUN LI $^{3}$, YUYUN ZHOU ${ }^{1}$, \\ XIAOHE WANG ${ }^{1}$, GUOJIAO FU ${ }^{1}$ and XUELING MA ${ }^{1}$

\begin{abstract}
${ }^{1}$ Department of Neurology, The Fourth Affiliated Hospital of Harbin Medical University, Harbin, Heilongjiang 150001;
${ }^{2}$ Department of Oncology, The First Hospital of Qiqihar, Qiqihar, Heilongjiang 161005; ${ }^{3}$ Department of Orthopedics, The First Affiliated Hospital of Harbin Medical University, Harbin, Heilongjiang 150001, P.R. China
\end{abstract}

Received May 23, 2017; Accepted September 12, 2017

DOI: 10.3892/etm.2017.5274

\begin{abstract}
The aim of this study was to investigate and analyze the effects of human umbilical cord blood mesenchymal stem cells (hUCB-MSCs) on the recovery of neurological function and telomerase reverse transcriptase (TERT) expression in brain tissue of rats with cerebral ischemia-reperfusion injury. A total of 100 healthy adult Wistar rats were randomly divided into two groups: The control group and the observation group according to the random number table method. After the model of cerebral ischemia-reperfusion injury was established, the rats in the observation group were treated with hUCB-MSCs $(10 \mathrm{ml} / \mathrm{kg})$, while the rats in the control group were treated with saline every day. The neurological deficit score and foot fault test were evaluated at 1,7 and 14 days after treatment, and the rats were sacrificed at 14 days to detect the expression of TERT in brain tissue. There was no significant difference in the scores of mNSS between the two groups before the model establishment $(\mathrm{P}>0.05)$, but there was significant differences in two groups after the operation $(\mathrm{P}<0.05)$. At 1 day after the operation, the mNSS score of the two groups peaked, which was decreased in the groups with the progress of treatment. The degree of decline in the observation group was significantly greater than that in the control group $(\mathrm{P}<0.05)$. Similarly, there was no significant difference in the number of errors between the two groups before the model establishment $(\mathrm{P}>0.05)$, but there was significant difference in two groups after the operation $(\mathrm{P}<0.05)$. At 1 day after the operation, the number of errors also peaked, which was reduced in the groups with the progress of treatment. The degree of reduction in the observation group was significantly greater than that in the control group $(\mathrm{P}<0.05)$.
\end{abstract}

Correspondence to: Dr Xueling Ma, Department of Neurology, The Fourth Affiliated Hospital of Harbin Medical University, 37 Yiyuan Street, Nangang, Harbin, Heilongjiang 150001, P.R. China

E-mail: gys6du@163.com

Key words: hUCB-MSCs, cerebral ischemia-reperfusion injury, telomerase reverse transcriptase, neurological recovery
The results of H\&E staining showed it had positive reaction as nucleus or cytoplasm stained brown or yellowish brown in the observation group, while it showed neuronal shrinkage, cytoplasm and nucleus yellow dye deepening in the control group as the significant positive reaction. The gray level of the TERT protein in the brain tissue of the control group was $0.458 \pm 0.052 \mathrm{LOD}$, which was significantly lower than that in the observation group with $0.983 \pm 0.056 \mathrm{LOD}(\mathrm{P}<0.05)$. In conclusion, hUCB-MSCs can effectively improve the neurological function and the expression of TERT in brain tissue of rats with cerebral ischemia-reperfusion injury, which may be helpful to reduce the ischemia-reperfusion injury of brain tissue.

\section{Introduction}

With the incidence of ischemic encephalopathy gradually increased, people have payed increased attention to the prevention and treatment of cerebral ischemia-reperfusion injury $(1,2)$. Cerebral infarction is a common cause of ischemic encephalopathy, thrombolytic therapy after cerebral infarction in time can save the lives of patients, but also may cause ischemia-reperfusion injury. Therefore, if the mechanism of ischemia-reperfusion injury is clear, it is of great significance for timely target treatment for ischemia-reperfusion injury. In recent years, there were more studies on ischemia-reperfusion injury, but there is still not a unified opinion on the prevention and treatment of ischemia-reperfusion injury. Studies showed that the expression of telomerase was increased in the brain tissues of patients with ischemia-reperfusion injury $(2,3)$, while some researchers found that telomerase was overexpressed after the injury of brain tissue, considering the protective effect of telomerase on ischemia-reperfusion injury in order to reduce the tissue damage (4). Human umbilical cord blood mesenchymal stem cells (hUCB-MSCs) can secrete a variety of cytokines and neurotrophic factors and promote nerve regeneration, but the conventional route of administration can not fully play the role of hUCB-MSCs. So some scholars proposed nasal administration of hUCB-MSCs to improve neurological function in rats with ischemia-reperfusion injury (5). At present, the study of hUCB-MSCs on nerve injury in rats with 
ischemia-reperfusion injury is scarce. So the aim of our study was to investigate the effects of hUCB-MSCs on rats with ischemia-reperfusion injury, in order to explore the mechanism of the prevention and treatment of ischemia-reperfusion injury.

\section{Materials and methods}

Animals. Experiments were performed using 100 healthy adult Wistar rats weighing 180-220 g, provided by College Animal Center, Harbin Medical University. Rats were kept in the animal house under constant temperature $\left(18-25^{\circ} \mathrm{C}\right)$ and humidity (60 to $70 \%$ ) on natural light with C060 sterilized commercial feed and free water intake, with $12 \mathrm{~h}$ before fasting. The study was approved by the Ethics Committee of Harbin Medical University.

Modeling method. All rats were randomly divided into control group and observation group and treated with $10 \%$ chloral hydrate $(3.5 \mathrm{ml} / \mathrm{kg})$ at RT. After anesthesia, the middle cerebral artery ischemia-reperfusion model was established by the suture method, and the specific modeling method was according to the method given by Chopp and Li (6). Then the rats in the observation group were treated with hUCB-MSCs $(10 \mathrm{ml} / \mathrm{kg})$ by nasal administration for 14 days, with the saline in the control group. The neurological deficit score and foot fault test were evaluated at 1, 7 and 14 days after treatment, and the rats were sacrificed at 14 days to detect the expression of telomerase reverse transcriptase (TERT) in brain tissue.

The source of hUCB-MSCs: $30 \mathrm{ml}$ human umbilical cord blood was taken under sterile conditions with heparin anticoagulant, then transferred into a $15 \mathrm{ml}$ centrifuge tube and centrifuged at 2,800 x $\mathrm{g}$ for $20 \mathrm{~min}$. The upper plasma was removed, collecting the white blood cells in interface layer, and stored at $4^{\circ} \mathrm{C}$. The expression of TERT was detected by western blot analysis. The above operations are carried out by the researchers in our laboratory according to the instructions, and the reagents and kits were purchased from LanPai Biotechnology Co., Ltd. (Shanghai, China).

\section{Observation and evaluation of indicators}

The criteria for $m N S S$ (7): The four aspects as sensory, motor, reflex and muscle tensionin of rats were scored with the total score of 18. The higher the score, the more serious the nerve damage.

Foot fault test: The foot fault test was used to evaluate the disorder of rat forelimb, placing the rats on the Hanging mesh grid $(2.5 \times 2.5 \mathrm{~cm})$, then recording the total number of bilateral forelimb movement and the number of left forelimb foot errors. The percent of the number of left forelimb foot errors to the total number of bilateral forelimb movement as the statistical results.

Statistical analysis. The statistical analysis was performed with SPSS 21.0 (IBM Corp., Armonk, NY, USA) and the obtained data are in normal distribution. Measurement data were expressed as mean \pm SD and the t-test was performed. Counting data were calculated by Chi-square test. A P-value $<0.05$ was considered as a significant difference.

\section{Results}

Comparison of neurological deficit score between the two groups. There was no significant difference in the scores of mNSS between the two groups before the model establishment ( $P>0.05)$, but after the model established there was significant difference in two groups respectively $(\mathrm{P}<0.05)$. At 1 day after the operation, the mNSS score of two groups was peaked, which was decreased in the groups with the progress of treatment. The degree of decline in the observation group was significantly greater than that in the control group $(\mathrm{P}<0.05$; Table I).

Comparison of foot fault number between the groups. There was no significant difference in the foot fault number between the two groups before ischemia-reperfusion model establishment $(\mathrm{P}>0.05)$, but after the model established the foot fault frequency was significantly increased in each group. At 1 day after operation, the number of foot errors peaked, but it was decreased in the groups with the progress of treatment. The number of foot errors was decreased significantly in the observation group compared to that in the control group $(\mathrm{P}<0.05$; Table II).

The gray value of TERT content in brain tissue in the two groups. The gray value of TERT content in the observation group was $0.983 \pm 0.056$ LOD, which was significantly higher than that in the control group with the gray value of $0.458 \pm 0.052$ LOD $(\mathrm{P}<0.05$; Table III).

Comparison of groups of rats with brain tissue immunohistochemical reaction. The H\&E staining showed the nucleus or cytoplasm in the brain tissue of rats was brown or yellow brown in the observation group, which showed positive reaction (Fig. 1A). The cytoplasm and the nucleus were stained yellow in the control group, suggested the neurons were shrunken (Fig. 1B).

TERT protein expression in two groups. As Fig. 2 shows, the expression of TERT protein in brain tissue of rats in observation group was significantly decreased compared to that in the control group.

\section{Discussion}

Ischemia and hypoxia is an important factor for tissue necrosis. For the brain tissue, short-term ischemia and hypoxia can cause irreversible tissue damage (8). In recent years, with the deep study of ischemic diseases, increased number of studies found that ischemia is not the only factor for the tissue damage (9). Free radical release caused by ischemia-reperfusion plays an important role in the tissue injury induced by ischemia and hypoxia, which is known as ischemia-reperfusion injury. Telomerase refers to an enzyme that is responsible for telomere elongation in cells and is a basic nucleoprotein reverse transcriptase that binds telomere DNA to the eukaryotic chromosome ends. The primary role of telomerase is to maintain telomere integrity and prevents its loss in cell division (10). The activity of telomerase is inhibited in normal cells, but it can be significantly activated in tumor 
Table I. Neurological deficit score in two groups (mean \pm SD).

\begin{tabular}{|c|c|c|c|c|c|}
\hline \multirow[b]{2}{*}{ Groups } & \multirow[b]{2}{*}{$\mathrm{n}$} & \multicolumn{4}{|c|}{ mNSS score } \\
\hline & & Preoperative & Postoperative day 1 & Postoperative day 7 & Postoperative day 14 \\
\hline Control group & 50 & $0.56 \pm 0.12$ & $11.51 \pm 0.56^{\mathrm{a}}$ & $8.52 \pm 0.84^{\mathrm{a}}$ & $6.85 \pm 1.25^{\mathrm{a}}$ \\
\hline Observation group & 50 & $0.58 \pm 0.14$ & $10.54 \pm 0.58^{\mathrm{a}}$ & $4.59 \pm 0.68^{\mathrm{a}, \mathrm{b}}$ & $3.25 \pm 0.52^{\mathrm{a}, \mathrm{b}}$ \\
\hline Group & \multicolumn{5}{|c|}{$\mathrm{F}=12.32, \mathrm{P}=0.002$} \\
\hline Time & \multicolumn{5}{|c|}{$\mathrm{F}=8.12, \mathrm{P}=0.026$} \\
\hline Time group & \multicolumn{5}{|c|}{$\mathrm{F}=9.15, \mathrm{P}=0.012$} \\
\hline
\end{tabular}

${ }^{\text {a }}<<0.05$ compared with the preoperative score; ${ }^{b} \mathrm{P}<0.05$ compared with control group.

Table II. The number of foot errors in the two groups (mean \pm SD).

Wrong steps

\begin{tabular}{|c|c|c|c|c|c|}
\hline \multirow[b]{2}{*}{ Group } & \multirow[b]{2}{*}{$\mathrm{n}$} & \\
\hline & & Preoperative & Postoperative day 1 & Postoperative day 7 & Postoperative day 14 \\
\hline Control group & 50 & $0.44 \pm 0.15$ & $29.5 \pm 3.82^{\mathrm{a}}$ & $23.65 \pm 3.43^{\mathrm{a}}$ & $16.98 \pm 2.68^{\mathrm{a}}$ \\
\hline Observation group & 50 & $0.42 \pm 0.12$ & $28.32 \pm 3.46^{\mathrm{a}}$ & $20.32 \pm 3.24^{\mathrm{a}, \mathrm{b}}$ & $6.52 \pm 1.87^{\mathrm{a}, \mathrm{b}}$ \\
\hline Group & \multicolumn{5}{|c|}{$\mathrm{F}=10.45, \mathrm{P}=0.002$} \\
\hline Time & & \multicolumn{4}{|c|}{$\mathrm{F}=7.43, \mathrm{P}=0.034$} \\
\hline Time group & & \multicolumn{4}{|c|}{$\mathrm{F}=8.65, \mathrm{P}=0.027$} \\
\hline
\end{tabular}

${ }^{\mathrm{a}} \mathrm{P}<0.05$ compared with preoperative foot errors; ${ }^{\mathrm{b}} \mathrm{P}<0.05$ compared with control group.
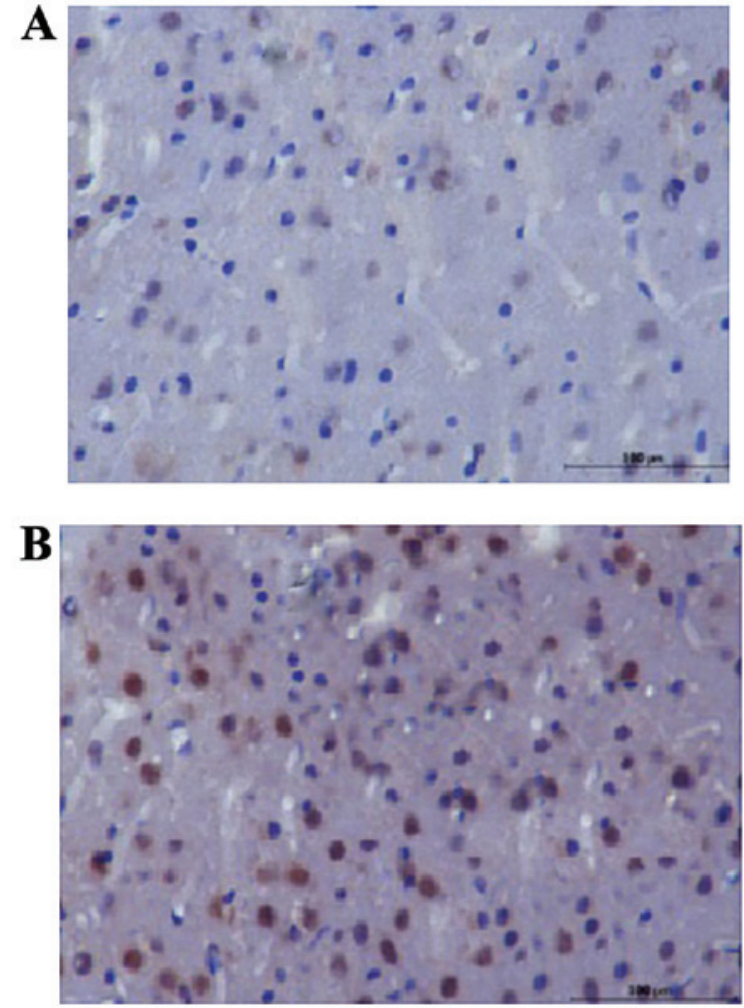

Figure 1. H\&E staining of the rat brain tissue immunohistochemical reaction. (A) The nucleus or cytoplasm was brown or yellow brown in the observation group, which showed positive reaction. (B) The cytoplasm and the nucleus were stained yellow in the control group, suggested the neurons were shrunken.
Table III. The gray value of TERT content in brain tissue of the two groups (mean $\pm \mathrm{SD}$ ).

\begin{tabular}{lcc}
\hline Group & $\mathrm{n}$ & TERT (WB, LOD) \\
\hline Control group & 50 & $0.458 \pm 0.052$ \\
Observation group & 50 & $0.983 \pm 0.056$ \\
T value & & 8.62 \\
P-value & & $<0.05$ \\
\hline
\end{tabular}

TERT, telomerase reverse transcriptase.

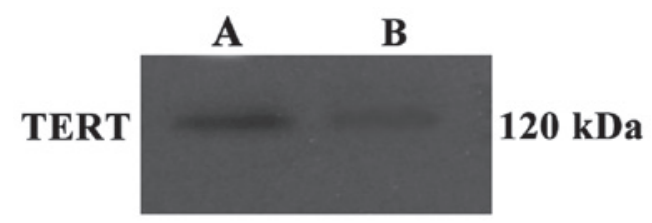

Figure 2. The expression of TERT protein in brain tissue of rats. A, the control group; B, the observation group; TERT, telomerase reverse transcriptase.

cells. TERT, as a catalytic subunit of telomerase, not only activates telomerase activity, but also protects mitochondria and has an anti-apoptotic effect. Studies showed that TERT can effectively improve neuronal apoptosis and neurological function in patients with ischemia-reperfusion injury (11). Stem cell therapy is a more popular treatment in recent years, 
which have a good therapeutic effect for some diseases. Some scholars have found that hUCB-MSCs contains a variety of cytokines and nerve growth factors, which can effectively improve neuronal damage, but these growth factors can not pass through the blood-brain barrier (12). Nasal administration not only allows drugs to enter the brain tissue, but also reduces the loss of stem cells.

In this study, there were no significant differences in the scores of mNSS between the two groups before the model establishment $(\mathrm{P}>0.05)$, but there was significant differences in two groups after the operation $(\mathrm{P}<0.05)$. At 1 day after the operation, the mNSS score of two groups peaked, which was decreased in the groups with the progress of treatment. The degree of decline in the observation group was significantly greater than that in the control group $(\mathrm{P}<0.05)$. Similarly, there were no significant differences in the number of preoperative footsteps between the two groups before the model establishment $(\mathrm{P}>0.05)$, but there was significant differences in two groups after the operation $(\mathrm{P}<0.05)$. At 1 day after the operation, the number of preoperative footsteps was peaked, which was reduced in the groups with the progress of treatment. The degree of reduction in the observation group was significantly greater than that in the control group $(\mathrm{P}<0.05)$. The results in our study showed that hUCB-MSCs could effectively reduce the neurological injury of rats with ischemia-reperfusion injury, which was consistent with the decrease of neuronal apoptosis in brain tissue. Zhao et al also found that after administration of hUCB-MSCs, neuronal dysfunction in rats with ischemia-reperfusion injury was improved, which was possibly related to the effects of TERT on promoting astrocyte proliferation and activation, increasing hypoxia ischemic rat brain astrocytes proliferation and promoting neuronal survival (13). With the gray value of the TERT protein as $0.458 \pm 0.052 \mathrm{LOD}$ in the control group, which was significantly lower than that in the observation group with $0.983 \pm 0.056 \mathrm{LOD}$, which suggested that hUCB-MSCs could effectively promote the expression of TERT in brain tissue of rats with ischemia-reperfusion. Chen et al also found that hUCB-MSCs significantly increased the expression of TERT protein in rat brain by nasal administration, possibly related to the effect of hUCB-MSCs on promoting the expression of TERT in brain tissue induced by cytokines and neurotrophic factors (14).

In conclusion, hUCB-MSCs can effectively improve the neurological function of cerebral ischemia-reperfusion injury in rats and improve the expression of TERT in brain tissue, which may help to reduce cerebral ischemia-reperfusion injury.

\section{Acknowledgements}

The study was supported by the Post-Doctoral Initial Fund of Heilongjiang Province (LBH-Q15091) and the Distinguished
Young Scholars Fund of the Fourth Clinical Medical College of Haerbin Medical University (HYDSYJQ201506).

\section{References}

1. Lahiani A, Zahavi E, Netzer N, Ofir R, Pinzur L, Raveh S, Arien-Zakay H, Yavin E and Lazarovici P: Human placental eXpanded (PLX) mesenchymal-like adherent stromal cells confer neuroprotection to nerve growth factor (NGF)-differentiated PC12 cells exposed to ischemia by secretion of IL- 6 and VEGF. Biochim Biophys Acta 1853: 422-430, 2015.

2. Yu H, Chen P, Yang Z, Luo W, Pi M, Wu Y and Wang L: Electroacupuncture at conception and governor vessels and transplantation of umbilical cord blood-derived mesenchymal stem cells for treating cerebral ischemia/reperfusion injury. Neural Regen Res 9: 84-91, 2014.

3. Guerrero-Beltrán CE, Tapia E, Sánchez-González DJ, MartínezMartínez CM, Cristobal-García M and Pedraza-Chaverri J: Tert-Butylhydroquinone pretreatment protects kidney from ischemia-reperfusion injury. J Nephrol 25: 84-89, 2012.

4. Khan I, Ali A, Akhter MA, Naeem N, Chotani MA, Iqbal H, Kabir N, Atiq M and Salim A: Epac-Rap1-activated mesenchymal stem cells improve cardiac function in rat model of myocardial infarction. Cardiovasc Ther 35: 35, 2017.

5. Karantalis V and Hare JM: Use of mesenchymal stem cells for therapy of cardiac disease. Circ Res 116: 1413-1430, 2015.

6. Chopp M and Li Y: Treatment of neural injury with marrow stromal cells. Lancet Neurol 1: 92-100, 2002.

7. Chen J, Sanberg PR, Li Y, Wang L, Lu M, Willing AE, Sanchez-Ramos J and Chopp M: Intravenous administration of human umbilical cord blood reduces behavioral deficits after stroke in rats. Stroke 32: 2682-2688, 2001.

8. Luo CJ, Zhang FJ, Zhang L, Geng YQ, Li QG, Hong Q, Fu B, Zhu F, Cui SY, Feng Z, et al: Mesenchymal stem cells ameliorate sepsis-associated acute kidney injury in mice. Shock 41: 123-129, 2014.

9. Sun T, Gao GZ, Li RF, Li X, Li DW, Wu SS, Yeo AE and Jin B: Bone marrow-derived mesenchymal stem cell transplantation ameliorates oxidative stress and restores intestinal mucosal permeability in chemically induced colitis in mice. Am J Transl Res 7: 891-901, 2015.

10. Chung DJ, Choi CB, Lee SH, Kang EH, Lee JH, Hwang SH, Han H, Lee JH, Choe BY, Lee SY and Kim HY: Intraarterially delivered human umbilical cord blood-derived mesenchymal stem cells in canine cerebral ischemia. J Neurosci Res 87: 3554-3567, 2009.

11. Li J, Qu Y, Chen D, Zhang L, Zhao F, Luo L, Pan L, Hua J and $\mathrm{Mu}$ D: The neuroprotective role and mechanisms of TERT in neurons with oxygen-glucose deprivation. Neuroscience 252: 346-358, 2013.

12. Leong KH, Zhou LL, Lin QM, Wang P, Yao L and Huang ZT: Therapeutic effects of various methods of MSC transplantation on cerebral resuscitation following cardiac arrest in rats. Mol Med Rep 13: 3043-3051, 2016.

13. Zhao F, Qu Y, Xiong T, Duan Z, Ye Q and Mu D: The neuroprotective role of TERT via an antiapoptotic mechanism in neonatal rats after hypoxia-ischemia brain injury. Neurosci Lett 515: 39-43, 2012.

14. Chen M,Xiang Z and Cai J: The anti-apoptotic and neuro-protective effects of human umbilical cord blood mesenchymal stem cells (hUCB-MSCs) on acute optic nerve injury is transient. Brain Res 1532: 63-75, 2013. 